\title{
Evaluation of $P$ wave Dispersion and Tissue Doppler Imaging for Predicting Paroxysmal Atrial Fibrillation in Patients with Hypertension
}

\author{
Xiaohui Zhang, ${ }^{1}$ Wei Zeng, ${ }^{2 *}$ Yuanmin Li, ${ }^{2}$ Dapeng Hou, ${ }^{3}$ Xiuchang Li, ${ }^{2}$ Wenbo Xu ${ }^{1}$ \\ ${ }^{1}$ Electrocardiographic Room, Affiliated Hospital of Taishan Medical University, PR China; ${ }^{2}$ Department of Cardiology, Affiliated \\ Hospital of Taishan Medical University, PR China; ${ }^{3}$ Intensive Care Unit, Affiliated Hospital of Taishan Medical University, PR China
}

\section{ABSTRACT}

Background: There are no previous studies dealing with paroxysmal atrial fibrillation (AF) and hypertension using electrocardiogram and tissue doppler imaging (TDI). The aim of this study was to investigate and identify the predictive indicators for paroxysmal AF in hypertensive patients using $\mathrm{P}$ wave dispersion $(\mathrm{Pd})$ and TDI.

Methods: Patients with hypertension were enrolled. Patients with paroxysmal AF were classified as the PAF group, and patients without a history of paroxysmal AF were classified as the NAF group. The clinical data, $\mathrm{P}$ wave indicators and TDI indicators were collected and compared between the two groups.

Results: A total of 120 patients were enrolled into the study with 40 cases in the PAF group and 80 cases in the NAF group. Compared with NAF group, $\mathrm{Pd}$, maximum $\mathrm{P}$ wave duration (Pmax), left ventricular end-diastolic dimension (LVEDd) and left atrial dimension (LAD) were significantly longer $(P<.05)$ in the PAF group. PAL, PAI, PAR, LR, LI and IR were significantly longer $(P<.05)$ in the PAF group than in the NAF group. As for ROC analysis, Pd and PAL had the greatest area under the curve. The best diagnostic value of $\mathrm{Pd}$ and PAL was $40 \mathrm{~ms}$ and $78 \mathrm{~ms}$, respectively. The combination of $\mathrm{Pd} \geq 40 \mathrm{~ms}$ with $\mathrm{Pmax} \geq 110 \mathrm{~ms}$ showed higher specificity and positive predictive value but decreased sensitivity and negative predictive value for paroxysmal AF.

Conclusions: The PAF group had significantly longer atrial electromechanical time and higher $\mathrm{Pd}$ compared with NAF group. The combination of $\mathrm{Pd}$ and TDI may be helpful to predict the onset of paroxysmal AF in patients with hypertension.

\section{INTRODUCTION}

Atrial fibrillation (AF) is the most common sustained cardiac arrhythmia [Hijazi 2013; Andrade 2014] and accounts for one-third of hospitalizations for rhythm disorders. Atrial

Received May 12, 2017; received in revised form fuly 10, 2017; accepted fuly $13,2017$.

Correspondence: Wei Zeng, Department of Cardiology, Affiliated Hospital of Taishan Medical University, No. 706 Taishan Street, Taian, Shandong Province 271000, P.R.China; 8618505386982; fax: 8605386229999 (email: zengwei7268@163.com). fibrillation is of public health importance and profoundly increases morbidity, mortality, and health-related expenditures [Khaji 2017]. Atrial fibrillation affects 33 million individuals worldwide, and increases the risk of stroke, heart failure and death, and also impairs quality of life [Chugh 2014]. The prevalence rate of AF is $0.77 \%$ in China with an increasing trend with old-age [Zhou 2004]. AF exerts a profound negative impact on the quality and quantity of life of millions [Piccini 2016].

The understanding of the etiology of AF has improved markedly, with structural and electrical remodeling of the left atrium being increasingly recognized as a process that precedes and contributes to AF vulnerability. Since elevated systemic pressures influence the size and function of the left atrium, uncontrolled hypertension is a key contributor to the generation of a substrate vulnerable to AF. Hypertension is the most common cardiovascular comorbidity among patients with AF. Hypertension leads to electrical and structural alterations to the left atrium that predisposes to AF. Epidemiologic studies have shown that hypertension is associated with a 1.8-fold increased risk of developing new-onset $\mathrm{AF}$ and a 1.5- fold increased risk of progression to permanent AF [de Vos 2010]. Up to now, there have been no studies showing that antihypertensive therapy can reduce AF burden. However, current studies have proven that effective treatment of hypertension may reduce the likelihood of developing AF by preventing atrial stretch from elevated ventricular filling pressures, atrial fibrosis, and extracellular collagen deposition, as well as through several other important mechanisms [Schneider 2010; Zhang 2012]. Although hypertension is not the most dangerous element among the risk factors of $\mathrm{AF}$, hypertension has become the most important factor due to its high morbidity rate [Healey 2003; Huxley 2011]. It is still unclear about the exact mechanism of AF caused hypertension. Therefore, it is clinically meaningful to predict AF in patients with hypertension, and further adopt preventive measures to avoid AF.

It is recognized that there is an atrial and/or interatrial conduction delay in patients with paroxysmal AF. Takahashi et al found that preoperative Tissue Doppler Imaging (TDI)derived atrial conduction time can predict postoperative AF in patients undergoing mitral valve surgery for mitral valve regurgitation [Takahashi 2016]. To our knowledge, there is no previous study dealing with paroxysmal AF and hypertension using electrocardiogram and TDI in patients with paroxysmal AF. The aim of this study was to investigate the predictive 
indicators for paroxysmal AF in hypertensive patients using $\mathrm{P}$ wave dispersion and TDI. The clinical data, $\mathrm{P}$ wave indicators and TDI indicators were investigated.

\section{PATIENTS AND METHODS}

\section{Patients}

The study was approved by the Ethics Committee of the Affiliated Hospital of Taishan Medical University and was conducted in accordance with the Helsinki declaration. Consecutive patients with hypertension were enrolled in the study. The diagnosis of hypertension was on the basis of a systolic blood pressure (SBP) of $140 \mathrm{mmHg}$ or more or a diastolic blood pressure (DBP) of $90 \mathrm{mmHg}$ or more, measured on at least 2 different occasions according to the WHO-ISH (World Health Organization-International Society of Hypertension) guidelines (WHO 2004). Patients were admitted to the hospital between July 2013 and September 2014. Diagnostic criteria for sinus rhythm were obvious $\mathrm{P}$ wave $(<0.12 \mathrm{~s})$ in the preoperative electrocardiogram, amplitude of $\mathrm{P}$ wave in the limb leads $<0.25 \mathrm{mV}$, amplitude of $\mathrm{P}$ wave in the precordial leads $<0.15 \mathrm{mV}$, upright on II and III avF lead, inversed on avR lead, Pr of 0.12-0.20s, and normal QRS and ST-T. Diagnostic criteria for AF were the absence of $\mathrm{P}$ waves, with disorganized electrical activity in their place, and irregular R-R intervals. Diagnostic criteria for paroxysmal $\mathrm{AF}$ were $\mathrm{AF}$ duration $\leq 7$ days that convert to sinus rhythm [Association 2007].

The inclusion criteria were patients who met the diagnostic criteria for hypertension: systolic pressure $\geq 140 \mathrm{mmHg}$,

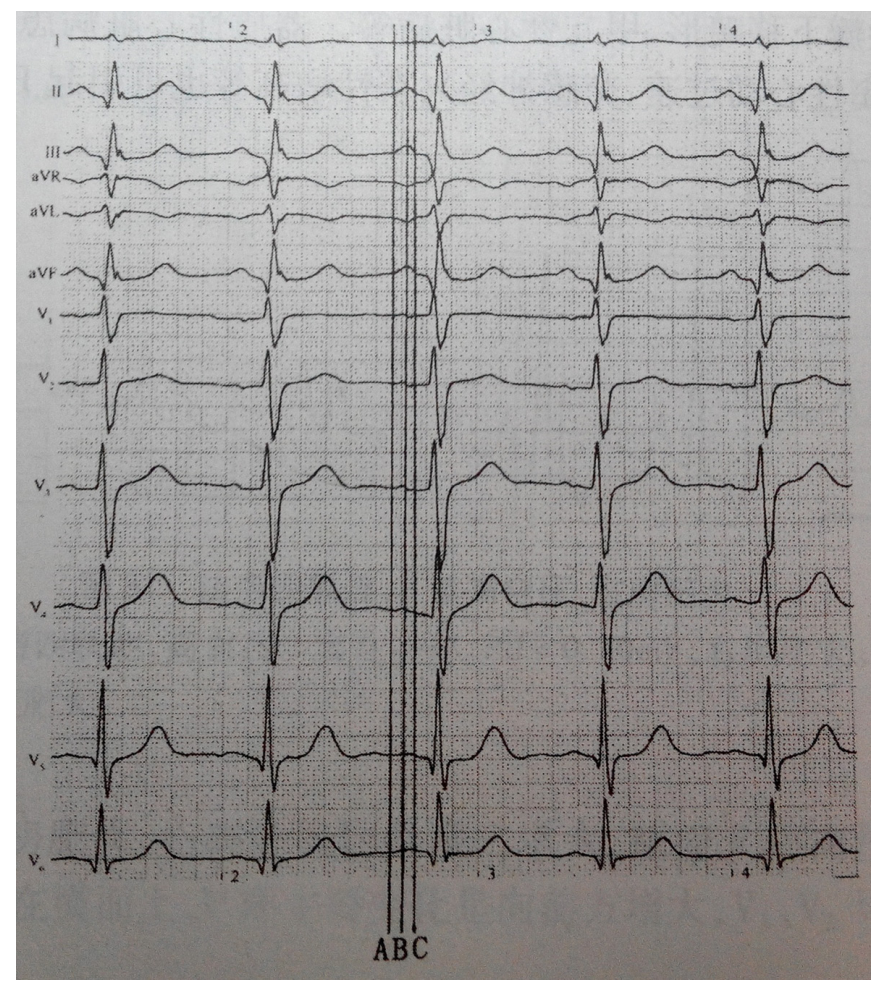

Figure 1. The measurement of $P$ wave dispersion. Line $A$ was the onset of $P$ wave; Line $B$ was the minimum $P$ wave duration (Pmin); Line $C$ was the maximum $P$ wave duration (Pmax). The distance between $B$ and $C$ was calculated and defined as $\mathrm{P}$ wave dispersion (Pd). In this case, Pd was $40 \mathrm{~ms}$. diastolic pressure $\geq 90 \mathrm{mmHg}$, paroxysmal AF confirmed by ECG, and more than two attacks of paroxysmal AF within the last 6 months. The exclusion criteria were: valvular heart disease, ischemic heart disease, corpulmonale, congenital heart disease, hyperthyroidism, secondary hypertension, electrolyte disorder and arrhythmia.

Clinical blood pressure in this study was measured three times by the traditional method with a mercury sphygmomanometer after the patient had rested for 30 minutes, and the mean values were obtained. Medical history of hypertension was recorded.

Table 1. Baseline Characteristics Between the Two Groups

\begin{tabular}{lccc}
\hline & $\operatorname{PAF}(\mathrm{n}=40)$ & $\mathrm{NAF}(\mathrm{n}=80)$ & $P$ \\
\hline Age & $68.70 \pm 10.48$ & $66.15 \pm 10.48$ & .212 \\
Sex (M/F) & $20 / 20$ & $38 / 42$ & .796 \\
Smoking $(\mathrm{Y} / \mathrm{N})$ & $12 / 28$ & $23 / 57$ & .887 \\
Alcohol drinking $(\mathrm{Y} / \mathrm{N})$ & $6 / 34$ & $15 / 65$ & .610 \\
Systolic arterial pressure & $154.50 \pm 30.46$ & $156.36 \pm 24.20$ & .717 \\
(mmHg) & & & \\
Diastolic arterial pressure & $96.15 \pm 18.36$ & $92.69 \pm 12.79$ & .231 \\
(mmHg) & $26 / 7 / 7$ & $53 / 16 / 11$ & .842 \\
GOH (1/2/3) & $318.35 \pm 115.27$ & $310.79 \pm 90.59$ & .699 \\
Uric acid (umol/L) & $71.41 \pm 32.75$ & $66.79 \pm 21.40$ & .362 \\
Creatinine (umol/L) & $5.93 \pm 2.33$ & $6.07 \pm 2.46$ & .768 \\
FBG (mmol/L) & $4.92 \pm 0.81$ & $4.96 \pm 1.51$ & .874 \\
Total cholesterol (mmol/L) & $1.28 \pm 0.69$ & $1.42 \pm 0.89$ & .379 \\
Triglyceride (mmol/L) & $2.48 \pm 0.64$ & $2.77 \pm 0.88$ & .072 \\
LDL (mmol/L) & $1.29 \pm 0.31$ & $1.28 \pm 0.31$ & .945 \\
HDL (mmol/L) & & & \\
\hline
\end{tabular}

M, male; F, female; $\mathrm{GOH}$, grade of hypertension; FBG, Fasting blood-glucose; LDL, low-density lipoprotein; HDL, high-density lipoprotein.

Table 2. Comparison of the Ultrasound Cardiogram Indexes Between the Two Groups

\begin{tabular}{lccc}
\hline & PAF $(\mathrm{n}=40)$ & $\operatorname{NAF}(\mathrm{n}=80)$ & $P$ \\
\hline Pmax (ms) & $125.5 \pm 26.7$ & $108.3 \pm 23.8$ & .001 \\
Pmin (ms) & $81.3 \pm 18.5$ & $80.2 \pm 21.5$ & .820 \\
Pd (ms) & $45.3 \pm 16.9$ & $27.5 \pm 19.3$ & $<.001$ \\
AO (mm) & $32.6 \pm 3.1$ & $31.6 \pm 3.1$ & .081 \\
LVPWd (mm) & $10.3 \pm 0.9$ & $10.1 \pm 1.1$ & .391 \\
IVSd (mm) & $10.6 \pm 1.1$ & $10.6 \pm 2.5$ & .990 \\
LVEDd (mm) & $49.8 \pm 5.9$ & $46.9 \pm 5.0$ & .005 \\
LAD (mm) & $42.2 \pm 5.1$ & $38.7 \pm 7.1$ & .005
\end{tabular}

PAF, paroxysmal atrial fibrillation; Pmax, maximum $\mathrm{P}$ wave duration; Pmin, minimum $P$ wave duration; $\mathrm{Pd}, \mathrm{P}$ wave dispersion; $\mathrm{AO}$ root, aortic root; LVPWd, Left ventricular posterior wall dimension at end diastolic; IVSd, interventricular septum dimension at end diastolic; LVEDd, Left ventricular end-diastolic dimension; LAD, left atrial dimension. 


\section{Methods for Electrocardiogram}

After the patient had rested in bed for 5 min, 12-lead synchronized ECG (F8322, Yasuo ltd. Co, Japan) was performed during sinus rhythm. The sweep speed was $50 \mathrm{~mm} / \mathrm{s}$ and voltage was $2 \mathrm{mV} / \mathrm{cm}$. At least five cardiac recycles were recorded, and the mean value was used. As for the dynamic ECG, 3M disposable electrode patches were pasted after cleaning the skin and the patient kept still. At least five cardiac cycles during the rest time were chosen for analysis. It was measured manually, and the average data were used.

For measurement of the $\mathrm{P}$ wave dispersion (Figure), line $\mathrm{A}$ was the onset of $\mathrm{P}$ wave, line $\mathrm{B}$ was the minimum $\mathrm{P}$ wave duration (Pmin), and line $\mathrm{C}$ was the maximum $\mathrm{P}$ wave duration (Pmax). The distance between $\mathrm{B}$ and $\mathrm{C}$ was defined as $\mathrm{P}$ wave dispersion (Pd) [Dilaveris 1998]. Pd was measured manually in more than three heart beats, and the average data were used. The criteria were $P \max \geq 110 \mathrm{~ms}$ and $\mathrm{Pd} \geq 40 \mathrm{~ms}$. The measurement was performed by an experienced doctor who was blind to the groups.

\section{Methods for TDI Indicators Measurements}

TDI images were reviewed by an experienced cardiovascular specialist with more than 10-years experience in echocardiography. Left ventricular posterior wall dimension at end diastolic (LVPWd), interventricular septum dimension at end diastolic (IVSd), Left ventricular end-diastolic dimension (LVEDd), early diastolic peak flow velocity (E), late diastolic peak flow velocity (A), the diameter of aortic root (AO) according to a previous report [Pieruzzi 2015]. In addition, the transverse diameter of the left ventricle, longitudinal diameter of the left ventricle, anteroposterior diameter of the left ventricle, and the left ventricle area were also measured. The time interval between the onset of $\mathrm{P}$ wave and the onset of A wave of tissue velocity imaging (TVI) was measured as P-A time [Rein 2003], corresponding to the atrial electromechanical time. The time interval between the onset of $\mathrm{P}$ wave and the onset of A wave of TDI in mitral annulus of the left ventricle lateral wall (PAL), the time interval between the onset of $\mathrm{P}$ wave and the onset of A wave of TDI in mitral

Table 3. The Comparison of the TDI Indicators Between the Two Groups

\begin{tabular}{lllc}
\hline & $\operatorname{PAF}(\mathrm{n}=40)$ & $\operatorname{NAF}(\mathrm{n}=80)$ & $P$ \\
$\operatorname{PAL}(\mathrm{mm})$ & $93.70 \pm 10.69$ & $76.60 \pm 14.07$ & $<.001$ \\
$\operatorname{PAl}(\mathrm{mm})$ & $70.78 \pm 11.56$ & $58.08 \pm 14.91$ & $<.001$ \\
$\operatorname{PAR}(\mathrm{mm})$ & $54.05 \pm 10.83$ & $43.79 \pm 12.24$ & $<.001$ \\
$\operatorname{LR}(\mathrm{mm})$ & $39.65 \pm 10.55$ & $32.81 \pm 7.03$ & $<.001$ \\
$\operatorname{LI}(\mathrm{mm})$ & $22.93 \pm 9.59$ & $18.53 \pm 5.16$ & .009 \\
IR $(\mathrm{mm})$ & $18.58 \pm 8.00$ & $14.29 \pm 9.29$ & .010 \\
\hline
\end{tabular}

PAL, the time interval between the onset of $P$ wave and the onset of $A$ wave of TDI in mitral annulus of left ventricle lateral wall; PAI, the time interval between the onset of $\mathrm{P}$ wave and the onset of $\mathrm{A}$ wave of $\mathrm{TVI}$ in mitral annulus of interventricular septum; PAR, the time interval between the onset of $P$ wave and the onset of $A$ wave of $T V I$ in tricuspid annulus of right ventricle; $L R$, the differences between the time intervals (PAL and time intervals (PAR); $L I$, the differences between the time intervals (PAL) and time intervals (PAI); IR, the differences between the time intervals (PAI) and time intervals (PAR). annulus of the interventricular septum (PAI), and the time interval between the onset of $\mathrm{P}$ wave and the onset of $\mathrm{A}$ wave of TVI in tricuspid annulus of the right ventricle (PAR) were measured according to a previous report [Rein 2000]. LR was calculated as the difference between the time intervals (PAL) and time intervals (PAR); LI was calculated the difference between the time intervals (PAL) and time intervals (PAI). IR was calculated as the difference between the time intervals (PAI) and time intervals (PAR).

\section{Statistical Analysis}

All data were analyzed using SPSS 19.0 (SPSS Inc, Chicago, USA). Qualitative data were presented as number or percentage. Quantitative data were presented as means \pm standard deviation (SD). The Kolmogorov-Smirnov test was used to test the normality of the distribution of the continuous variables. Comparison of the two groups for normally distributed data was performed with independent t-test. Logistic analysis and receiver operating characteristic (ROC) curve analysis were performed to evaluate the possible predictive parameters. $P<.05$ was considered statistically significant.

\section{RESULTS}

A total of 120 consecutive patients met the inclusion criteria and were enrolled into the study, with 40 cases in the PAF group and 80 cases in the NAF group. The baseline characteristics of the participants in the groups are described in Table 1 . There were no significant differences regarding the age, sex, smoking, alcohol drinking, systolic arterial pressure, diastolic arterial pressure, grade of hypertension, uric acid, creatinine, fasting blood-glucose, total cholesterol, triglyceride, low-density lipoprotein, and high-density lipoprotein in two groups (all $P>.05$ ).

Compared with NAF group, Pd, Pmax, LVIDd and left atrial dimension $(\mathrm{LAD})$ were significantly longer $(P<.05)$ in PAF group. There were no statistical differences $(P>.05)$ regarding AO, LVWP and IVST between the two groups (Table 2). PAL, PAI, PAR, LR, LI and IR were significantly longer $(P<.05)$ in PAF group than in NAF group (Table 3$)$. Multivariate analysis showed that Pd and PAL were significant influencing factors (Table 4).

With $\mathrm{AF}$ as state variable and Pmax, Pd, LVDd, LAD, PAL, PAI, PAR, LR, LI and IR as test variables, a ROC curve was made and the area under and the curve was calculated. The results showed that Pd and PAL had the greatest area under the curve. The best diagnostic values of Pd and PAL

Table 4. Multivariate Analysis Logistic Regression Analysis of Ultrasound Cardiogram and TDI Indexes Between the Two Groups

\begin{tabular}{lcccc}
\hline Parameters & Significance & Exp (B) & \multicolumn{2}{c}{$95.0 \%$ Cl for Exp (B) } \\
& & Lower & Upper \\
\hline Pd & 0.007 & 1.138 & 1.036 & 1.249 \\
PAL & $<0.001$ & 1.147 & 1.081 & 1.217 \\
\hline
\end{tabular}

Pd, $\mathrm{P}$ wave dispersion; PAL, the time interval between the onset of $\mathrm{P}$ wave and the onset of A wave of TDI in mitral annulus of left ventricle lateral wall. 
Table 5. The Diagnostic Value of Pd > 40ms, PAL > 78ms and Their Combination

\begin{tabular}{lcccccccc}
\hline & AUC & $P$ & Sensitivity & Specificity & Positive Predictive Value & Negative Predictive Value & Kappa value & $P$ \\
\hline Pd & 0.791 & $<.001$ & $82.5 \%$ & $73.75 \%$ & $61.11 \%$ & $89.39 \%$ & 0.517 & $<.001$ \\
PAL & 0.810 & $<.001$ & $97.44 \%$ & $66.25 \%$ & $58.46 \%$ & $98.15 \%$ & 0.530 & $<.001$ \\
Pd + PAL & NS & NS & $77.50 \%$ & $91.25 \%$ & $81.58 \%$ & $89.02 \%$ & 0.696 & $<.001$ \\
\hline
\end{tabular}

Kappa values and $P$ values were calculated on the basis of $P d>40 m$ s and PAL $>78 m s ; P d, P$ wave dispersion; PAL, the time interval between the onset of $P$ wave and the onset of A wave of TDI in mitral annulus of left ventricle lateral wall; AUC, area under the curve; NS, not applicable

were $40 \mathrm{~ms}$ (sensitivity: 0.825 , specificity: 0.738 , Youden index: 0.563 ) and $78 \mathrm{~ms}$ (sensitivity: 0.950 , specificity: 0.663 , Youden index: 0.613) respectively. The Kappa values are shown in Table 5 .

\section{DISCUSSION}

The present study showed that there were statistical differences regarding $\mathrm{P}$ wave indicators and TDI indicators between the PAF group and the NAF group. TDI was valuable for predicting paroxysmal AF.

Electrical conduction delays in the atrium and ventricle is likely to cause AF [Buxton 1981]. The characteristics of the $\mathrm{P}$ wave may be useful for predicting AF. The present study showed that Pd, Pmax, IVLDd and LAD were significantly longer $(P<.05)$ in PAF group than those in NAF group. Pmax could be considered as a predictor for paroxysmal AF. As for Pmin, there was no statistical difference in two groups, which was consistent with a previous report by Ciaroni et al [Ciaroni 2000]. Dilaveris et al proposed that Pd was a specific and sensitive predictor for $\mathrm{AF}$ and can be used to evaluate the electrical conduction heterogeneity in the atrium, which can obviously increase the accuracy of Pmax as a predictor for AF. The present study showed that $\mathrm{Pd}$ is a sensitive predictor for paroxysmal $\mathrm{AF}$ after multivariate logistic regression analysis. Ciaroni et al [Ciaroni 2000] and Guo et al [Guo 1999] reported that $\mathrm{Pd}$ could be used as an independent predictor for $\mathrm{AF}$, while was contrary to previous claims by Dilaveris et al [Dilaveris 2000].

The different results among the above reports might be due to the different measurement methods that were used. Manual measurement was adopted in the present study, which was consistent with the previous report by Ciaroni et al. Taking manual measurements will inevitably cause measurement errors because of the start point and endpoint determination. To minimize the error caused by manual measurement, $\mathrm{P}$ wave width was measured in five consecutive cardiac cycles by the same physician, with the average values used for final analysis. In addition, a 12-lead synchronous resting ECG was recorded, and the width of $\mathrm{P}$ wave was also measured. Thus, this reduced the measurement errors and ensured the objectivity of $\mathrm{P}$ wave. Errors may occur when only II-lead and V1- lead P waves are used to determine Pmax, Pmin and Pd [Yuan 2003].

Drugs can also exert an influence on the results, such as calcium antagonist, angiotensin converting enzyme inhibitors (ACEI) and blockers of beta receptors [Li 2001].

The possible mechanism may be that the activation of the renin-angiotensin-aldosterone system in hypertensive patients could prompt and maintain the AF. Ciaroni et al
[Ciaroni 2000] found that systolic blood pressure and age are important independent risk factors for $\mathrm{AF}$ in patients with hypertension. However, our study did not show the correlation of the above factors. This might be attributed to the limited samples.

As we know, atrial myocardial wall is thin with poor tension and thus vulnerable to geometric configuration changes under the influence of various pathological factors and mechanical forces. The atrial expansion and aggravated fibrosis in patients with primary hypertension can lead to diffuse atrial electrical activity and slow conduction velocity, which then increases the atrial electrophysiological anisotropy, eventually resulting in the occurrence of AF. The focal fibrosis of the atrial myocardium aggravates the heterogeneity of atrial electrical activity which increases the difference in atrial autorhythmicity and excitability among different parts of the atrium. Therefore, a larger difference in $\mathrm{P}$ wave duration among different leads will be shown on 12-lead resting ECG [Tong 2013].

Andrikopoulos et al [Andrikopoulos 2000] suggested that the dispersion degree of the $\mathrm{P}$ wave has positive predictive value for $\mathrm{AF}$. However, there are still controversial results regarding the patients with primary hypertension and concomitant paroxysmal AF [Ciaroni 2000; Ozer 2000]. Guo et al [Guo 2007] reported that $\mathrm{Pd} \geq 40 \mathrm{~ms}$ and $\mathrm{Pmax} \geq 110 \mathrm{~ms}$ were reliable predictors for AF. In this study, our findings showed that $\mathrm{Pd} \geq 40 \mathrm{~ms}$ showed higher predictive value for paroxys$\mathrm{mal} \mathrm{AF}$ in patients with hypertension which is in accordance with the previous study [Wang 2005]. In addition, we also found that $P d \geq 40 \mathrm{~ms}, P \max \geq 110 \mathrm{~ms}$ and the combinations of these two indexes were reliable predictors for paroxysmal AF. The combination of $\mathrm{Pd} \geq 40 \mathrm{~ms}$ with $\mathrm{Pmax} \geq 110 \mathrm{~ms}$ showed higher specificity and positive predictive value but decreased sensitivity and negative predictive value for paroxysmal AF.

TDI is useful in early detection of myocardial dysfunction in several diseases and can give accurate information about the functions of the ventricle in hypertensive patients [Mori 2007; Tenekecioglu 2010]. TDI is sensitive and reliable when determining left ventricular hypertrophy. However, the limitation of pulse repetition frequency may produce frequency distortion. To avoid the above distortion, low frequency probe, reducing sampling depth, moving the zero line, changing the angle between the direction of acoustic beam and blood flow, and increasing pulse repetition frequency were adopted in this study.

\section{CONCLUSIONS}

The present study suggested that PAF group showed significantly longer atrial electromechanical time and higher 
Pd compared with NAF group. The combination of Pd and TDI may be helpful to predict the onset of paroxysmal AF in patients with hypertension.

\section{REFERENCES}

Andrade J, Khairy P, Dobrev D, Nattel S. 2014. The clinical profile and pathophysiology of atrial fibrillation: relationships among clinical features, epidemiology, and mechanisms. Circ Res 114:1453-1468.

Andrikopoulos GK, Dilaveris PE, Richter DJ, et al. 2000. Increased variance of $\mathrm{P}$ wave duration on the electrocardiogram distinguishes patients with idiopathic paroxysmal atrial fibrillation. Pacing Clin Electrophysiol 23:1127-1132.

Association EHR, Fuster V, Rydén LE, et al. 2007. ACC/AHA/ESC 2006 guidelines for the management of patients with atrial fibrillation-executive summary: a report of the American College of Cardiology/ American Heart Association Task Force on Practice Guidelines and the European Society of Cardiology Commi. Eur Heart J 59:1231-1265.

Buxton AE, Josephson ME. 1981. The role of $\mathrm{P}$ wave duration as a predictor of postoperative atrial arrhythmias. Chest 80:68-73.

Chugh SS, Havmoeller R, Narayanan K, et al. 2014. Worldwide Epidemiology of Atrial Fibrillation A Global Burden of Disease 2010 Study. Circulation 129:837-847.

Ciaroni S, Cuenoud L, Bloch A. 2000. Clinical study to investigate the predictive parameters for the onset of atrial fibrillation in patients with essential hypertension. Am Heart J 139:814-819.

de Vos CB, Pisters R, Nieuwlaat R, et al. 2010. Progression from paroxysmal to persistent atrial fibrillation clinical correlates and prognosis. $\mathrm{J}$ Am Coll Cardiol 55:725-731.

Dilaveris PE, Gialafos EJ, Sideris SK, et al. 1998. Simple electrocardiographic markers for the prediction of paroxysmal idiopathic atrial fibrillation. Am Heart J 135:733-738.

Dilaveris PE, Gialafos EJ, Andrikopoulos GK, et al. 2000. Clinical and Electrocardiographic Predictors of Recurrent Atrial Fibrillation. Pacing Clin Electrophysiol 23:352-358.

Guo JH. 1999. P wave dispersion. J Clin Electrocardiol 189-192.

Guo YP. Fang WH, Cheng S-T. 2007. Analyzing effects of P wave dispersion on paroxysmal atrial fibrillation. Chin J Prim Med Pharm $14: 411-412$

Healey JS, Connolly SJ. 2003. Atrial fibrillation: hypertension as a causative agent, risk factor for complications, and potential therapeutic target. American Journal of Cardiology 91:9-14.

Hijazi Z, Oldgren J, Siegbahn A, Granger CB, Wallentin L. 2013. Biomarkers in atrial fibrillation: a clinical review. Eur Heart J 34:1475-1480.

Huxley RR. 2011. Absolute and Attributable Risks of Atrial Fibrillation in Relation to Optimal and Borderline Risk Factors The Atherosclerosis Risk in Communities (ARIC) Study. Circulation 123:1501-1508.

Khaji A, Kowey PR. 2017. Update on atrial fibrillation. Trends Cardiovasc Med 27:14-25.
Li D, Shinagawa K, Pang L, et al. 2001. Effects of Angiotensin-Converting Enzyme Inhibition on the Development of the Atrial Fibrillation Substrate in Dogs With Ventricular Tachypacing induced Congestive Heart Failure. Circulation 104:2608-2614.

Mori K, Hayabuchi Y, Inoue M, et al. 2007. Myocardial strain imaging for early detection of cardiac involvement in patients with Duchenne's progressive muscular dystrophy. Echocardiography 24:598-608.

Ozer N, Aytemir K, Atalar E, et al. 2000. P wave dispersion in hypertensive patients with paroxysmal atrial fibrillation. Pacing Clin Electrophysiol 23:1859-1862.

Piccini JP, Fauchier L. 2016. Rhythm control in atrial fibrillation. The Lancet 388:829-840.

Pieruzzi F, Antolini L, Salerno FR, et al. 2015. The role of blood pressure, body weight and fat distribution on left ventricular mass, diastolic function and cardiac geometry in children. J Hypertens 33:1182-1192.

Rein A, O'Donnell C, Colan S, Sear-Brent L, Marx G. editors. 2000. Right and left atrial contraction time and electromechanical coupling using tissue velocity imaging. Circulation 383 .

Rein AJ, O'Donnell CP, Colan SD, Marx GR. 2003. Tissue velocity Doppler assessment of atrial and ventricular electromechanical coupling and atrioventricular time intervals in normal subjects. Am J Cardiol 92:1347-1350

Schneider MP, Hua TA, Böhm M, et al. 2010. Prevention of Atrial Fibrillation by Renin-Angiotensin System Inhibition : A Meta-Analysis. J Am Coll Cardiol 55:2299-2307.

Takahashi S, Katayama K, Watanabe M, et al. 2016. Preoperative Tissue Doppler Imaging-Derived Atrial Conduction Time Predicts Postoperative Atrial Fibrillation in Patients Undergoing Mitral Valve Surgery for Mitral Valve Regurgitation. Circ J 80:101-109.

Tenekecioglu E, Karabulut A, Yilmaz M. 2010. Comparison of tissue Doppler dynamics with Doppler flow in evaluating left atrial appendage function by transesophageal echocardiography in prehypertensive and hypertensive patients. Echocardiography 27:677-686.

Tong D. 2013. The relation between $\mathrm{P}$ wave dispersion and primary hypertension complicated with paroxysmal atrial fibrillation. Prevention and Treatment of Cardio-Cerebral-Vascular Disease 13:376-378.

Wang CQ, Zhou M, Zhan ZQ, et al. 2005. Predictive value of $P$ wave dispersion on paroxysmal atrial fibrillation in primary hypertensive patients. Clinical Focus 245-7.

Yuan YQ, Liu HL, Ma YX. 2003. The effect of the inhibitors of angiotensin converting enzyme on $\mathrm{P}$ wave dispersion in patients with organic heart disease and paroxysmal atrial fibrillation. J Clin Cardiol 690-691.

Zhang H, Xin-Zheng LU. 2012. Hypertension and Atrial Fibrillation:Diagnostic Approach,Prevention and Treatment. Position Paper of the Working Group 'Hypertension Arrhythmias and Thrombosis' of the European Society of Hypertension. Adv Cardiovasc Dis $30: 239-252$.

Zhou ZQ, Hu DY, Chen J, et al. 2004. [An epidemiological survey of atrial fibrillation in China]. Zhonghua Nei Ke Za Zhi 43:491-494. 